\title{
THE FIRST HYPERVELOCITY STAR FROM THE LAMOST SURVEY
}

\author{
Zheng Zheng $^{1}$, Jeffrey L. Carlin ${ }^{2}$, Timothy C. Beers ${ }^{3,4}$, Licai Deng $^{5}$, Carl J. Grillmair ${ }^{6}$, Puragra Guhathakurta ${ }^{7}$, \\ Sébastien Lépine ${ }^{8}$, Heidi Jo Newberg ${ }^{2}$, Brian Yanny ${ }^{9}$, Haotong Zhang $^{5},{\text { Chao } \text { Liv }^{5} \text {, Ge Jin }}^{10}$, and Yong Zhang ${ }^{11}$ \\ ${ }^{1}$ Department of Physics and Astronomy, University of Utah, UT 84112, USA; zhengzheng @ astro.utah.edu \\ ${ }^{2}$ Department of Physics, Applied Physics, and Astronomy, Rensselaer Polytechnic Institute, 110 8th Street, Troy, NY 12180, USA \\ ${ }^{3}$ National Optical Astronomy Observatory, Tucson, AZ 85719, USA \\ ${ }^{4}$ JINA: Joint Institute for Nuclear Astrophysics, University of Notre Dame, Notre Dame, IN 46556-5670, USA \\ ${ }^{5}$ National Astronomical Observatories, Chinese Academy of Sciences, Beijing 100012, China \\ ${ }^{6}$ Spitzer Science Center, 1200 East California Boulevard, Pasadena, CA 91125, USA \\ ${ }^{7}$ UCO/Lick Observatory, Department of Astronomy and Astrophysics, University of California, Santa Cruz, CA 95064, USA \\ ${ }^{8}$ Department of Physics and Astronomy, Georgia State University, 25 Park Place, Suite 605, Atlanta, GA 30303, USA \\ ${ }^{9}$ Fermi National Accelerator Laboratory, P.O. Box 500, Batavia, IL 60510, USA \\ ${ }^{10}$ University of Science and Technology of China, Hefei 230026, China \\ ${ }^{11}$ Nanjing Institute of Astronomical Optics \& Technology, National Astronomical Observatories, \\ Chinese Academy of Sciences, Nanjing 210042, China \\ Received 2014 January 20; accepted 2014 February 22; published 2014 April 2
}

\begin{abstract}
We report the first hypervelocity star (HVS) discovered from the LAMOST spectroscopic survey. It is a B-type star with a heliocentric radial velocity of about $620 \mathrm{~km} \mathrm{~s}^{-1}$, which projects to a Galactocentric radial velocity component of $\sim 477 \mathrm{~km} \mathrm{~s}^{-1}$. With a heliocentric distance of $\sim 13 \mathrm{kpc}$ and an apparent magnitude of $\sim 13 \mathrm{mag}$, it is the closest bright HVS currently known. With a mass of $\sim 9 M_{\odot}$, it is one of the three most massive HVSs discovered so far. The star is clustered on the sky with many other known HVSs, and its position suggests a possible connection to Galactic center structures. With the current poorly determined proper motion, a Galactic center origin of this HVS remains consistent with the data at the $1 \sigma$ level, while a disk runaway origin cannot be excluded. We discuss the potential of the LAMOST survey to discover a large statistical sample of HVSs of different types.
\end{abstract}

Key words: Galaxy: center - Galaxy: disk - Galaxy: halo - Galaxy: kinematics and dynamics - stars: early-type stars: individual (J091206.52+091621.8)

Online-only material: color figures

\section{INTRODUCTION}

Hypervelocity stars (HVSs) are stars with velocities that exceed the escape velocity of the Galaxy. They were first predicted by Hills (1988) as a consequence of the tidal disruption of tight binary stars by the central massive black hole (MBH) of the Galaxy. Since the first discovery of an HVS (Brown et al. 2005), around 20 HVSs have been found (Edelmann et al. 2005; Hirsch et al. 2005; Brown et al. 2006a, 2006b, 2007a, 2007b, 2012). Here we report the discovery of the first HVS in the Large Sky Area Multi-Object Fiber Spectroscopic Telescope (LAMOST) survey.

Besides the Hills mechanism, HVSs may also be produced by the interaction among single stars and an intermediate-mass black hole inspiralling toward the central MBH (Yu \& Tremaine 2003), as well as the tidal disruption of dwarf galaxies (Abadi et al. 2009). Some HVSs may be runaway stars (Blaauw 1961), e.g., as the surviving companion stars in the white dwarf + helium star channel of Type Ia supernovae (Wang \& Han 2009), or from interactions among multiple stars (e.g., Gvaramadze et al. 2009; Tutukov \& Fedorova 2009).

HVSs provide a unique probe for a wide range of Galactic science (Kenyon et al. 2008), on scales from a few parsecs (near the central MBH) to $\sim 10^{5} \mathrm{pc}$ (the Galactic halo). The spatial and velocity distributions, as well as the detection frequencies of HVSs, can be used to test the ejection mechanisms. If HVSs are ejected from the Galactic center (GC), the number density, velocity, and stellar type distributions of HVSs can reveal the environment around the central $\mathrm{MBH}$ and the stellar mass distribution near the MBH (e.g., Brown et al. 2006a; Kollmeier
\& Gould 2007; Lu et al. 2007; Kollmeier et al. 2010). The sky distribution of HVSs suggests a connection to the $\mathrm{S}$ stars in the two disks near the central MBH (e.g., Lu et al. 2010), which may provide clues to the MBH growth (Bromley et al. 2012). The trajectories of HVSs can also be used to probe the shape of the dark matter halo of the Galaxy (Gnedin et al. 2005; Yu \& Madau 2007).

For all of the above applications, it is desirable to assemble a large statistical sample of HVSs. The LAMOST survey has this potential, as described below. In this Letter, we report the first HVS discovered in the internal Data Release 1 (DR1) of LAMOST. In Section 2, we provide a brief description of the data, and thereafter focus on the properties of the HVS and discuss their implications. Finally, in Section 3, we summarize the results and forecast the prospects of further HVS discoveries from the LAMOST survey.

\section{THE LAMOST SURVEY AND ITS FIRST HVS}

\subsection{Data}

LAMOST is a $4 \mathrm{~m}$ Schmidt telescope (now called the Guo Shoujing Telescope) at the Xinglong Observing Station of the National Astronomical Observatories of China. It is equipped with 4000 optical fibers in the focal plane, taking spectra with resolution $R=\lambda / \Delta \lambda=1800$. Within the LAMOST spectroscopic survey (Cui et al. 2012; Zhao et al. 2012), the LAMOST Experiment for Galactic Understanding and Exploration (LEGUE; Deng et al. 2012) aims to take $\sim 8$ million stellar spectra for targets covering $16,000 \mathrm{deg}^{2}$ of sky over the course of five years. The target-selection criteria required to 
Table 1

Properties of LAMOST-HVS1

\begin{tabular}{ll}
\hline \hline & \multicolumn{1}{c}{$\mathrm{J} 091206.52+091621.8$} \\
\hline Position $(J 2000)$ & $(\alpha, \delta)=(138.027199,9.272725)$ \\
& $(l, b)=(221.099564,35.407261)$ \\
Magnitudes & $g=12.91 r=13.22 i=13.50$ \\
& $B=12.96 \mathrm{~V}=13.06 \mathrm{~J}=13.36$ \\
& $H=13.43 \mathrm{~K}=13.53$ \\
Distance & $13.4 \pm 2.2 \mathrm{kpc}(\mathrm{Heliocentric})$ \\
& $19.4 \pm 2.1 \mathrm{kpc}(\mathrm{Galactocentric})$ \\
Radial velocity & $v_{r \odot}=620 \pm 10 \mathrm{~km} \mathrm{~s}^{-1}$ \\
& $v_{\mathrm{rf}}=477 \pm 10 \mathrm{~km} \mathrm{~s}^{-1}$ \\
Proper motion & $\left(\mu_{\alpha} \mathrm{cos} \delta, \mu_{\delta}\right)$ \\
$\quad\left(\right.$ mas yr $\left.^{-1}\right)$ & $(-4.0 \pm 0.7,-4.9 \pm 1.2)$ [UCAC4] \\
& $(-2.5 \pm 1.9,-1.2 \pm 1.9)$ [PPMXL] \\
Spectral type & $(0.9 \pm 1.9,0.9 \pm 1.9)[\mathrm{cPPMXL}]$ \\
$T_{\text {eff }}$ & $\mathrm{B}$ \\
$\log \left[g /\left(\mathrm{cm} \mathrm{s}^{-2}\right)\right]$ & $(2.07 \pm 0.12) \times 10^{4} \mathrm{~K}$ \\
{$[$ Fe/H] } & $3.67 \pm 0.19$ \\
Mass & $-0.13 \pm 0.07$ \\
\hline
\end{tabular}

achieve various science goals can be found in Carlin et al. (2012), Chen et al. (2012), Yang et al. (2012), and Zhang et al. (2012).

The internal DR1 of LAMOST spectra and stellar parameters includes spectra obtained during a pilot survey (from 2011 October to 2012 June; Luo et al. 2012) and the first year of the regular survey (from 2012 September). Most of the observed stars are brighter than $r=16$. We performed a systematic search for HVSs in the catalog, including stars of A-type or earlier; of these stars, one of the $\sim 10^{5}$ stars turned out to be a HVS (hereafter, denoted LAMOST-HVS1).

\subsection{Properties of LAMOST-HVS1}

The star (J091206.52+091621.8; LAMOST-HVS1) has been observed twice by LAMOST, separated by about 70 days (2012 December 23 and 2013 March 5). It is a bright star with magnitude around 13. The radial velocities at the two epochs from spectral fitting are consistent with each other within the uncertainties, and therefore there is no evidence of it being a close binary system.

The measured heliocentric radial velocity, $v_{r \odot}=620 \pm$ $10 \mathrm{~km} \mathrm{~s}^{-1}$, translates to a Galactocentric radial component $v_{\text {rf }}=477 \pm 10 \mathrm{~km} \mathrm{~s}^{-1}$, according to

$$
v_{\mathrm{rf}}=v_{r \odot}+U_{0} \cos l \cos b+\left(V_{\mathrm{LSR}}+V_{0}\right) \sin l \cos b+W_{0} \sin b,
$$

where we adopt $V_{\mathrm{LSR}}=250 \mathrm{~km} \mathrm{~s}^{-1}$ for the velocity of the local standard of rest (LSR; Reid et al. 2009; McMillan \& Binney 2010) and $\left(U_{0}, V_{0}, W_{0}\right)=(11.1,12.24,7.25) \mathrm{km} \mathrm{s}^{-1}$ for the peculiar motion of the Sun with respect to the LSR (Schönrich et al. 2010). The star has proper motion measurements $\left(\mu_{\alpha} \cos \delta, \mu_{\delta}\right)=(-4.0 \pm 0.7,-4.9 \pm 1.2)$ mas yr $^{-1}$ in the UCAC4 catalog (Zacharias et al. 2013) and ( $-2.5 \pm 1.9,-1.2 \pm$ 1.9) $\mathrm{mas}^{-1} \mathrm{r}^{-1}$ in the PPMXL catalog (Roeser et al. 2010). We find that quasars within two degrees around the star in the PPMXL catalog have a net proper motion, and if we correct for this systematic offset, the proper motion of the star becomes $(0.9 \pm 1.9,0.9 \pm 1.9)$ mas $^{-1} r^{-1}$ (listed as cPPMXL in Table 1$)$. A more accurate determination is clearly desirable.

Figure 1 shows the low-resolution LAMOST spectrum of the star. The basic stellar atmospheric parameters, listed in

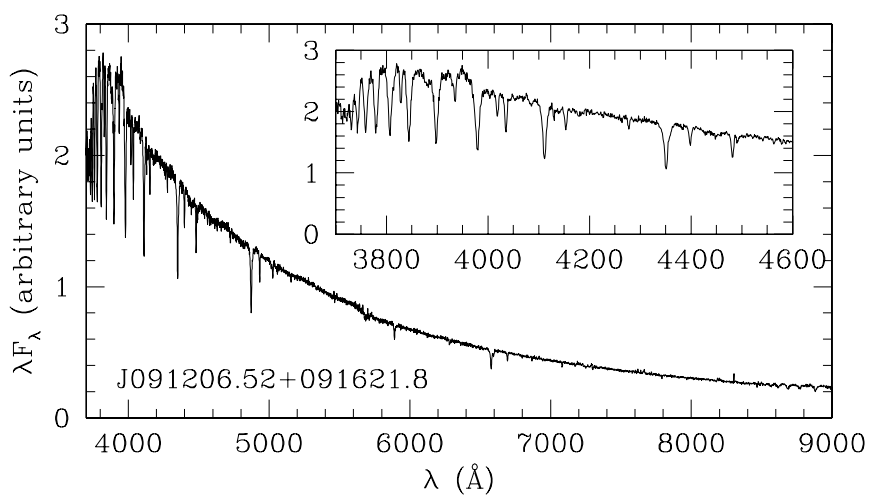

Figure 1. Spectrum of LAMOST-HVS1 taken with the Guo Shoujing Telescope. The inset shows a close-up view of the blue end of the spectrum.

Table 1, are obtained by fitting the spectrum with the University of Lyon Spectroscopic analysis Software (ULySS ${ }^{12}$; Koleva et al. 2009). It has an effective temperature $T_{\text {eff }} \simeq 2 \times 10^{4} \mathrm{~K}$, surface gravity $\log \left[\mathrm{g} /\left(\mathrm{cm} \mathrm{s}^{-2}\right)\right] \simeq 3.7$, and slightly sub-solar metallicity, $[\mathrm{Fe} / \mathrm{H}] \simeq-0.13$. The presence of strong helium lines indicates that it is an early B-type star.

To determine the distance and mass of LAMOST-HVS1, we make use of the list of masses and luminosities of B-type stars compiled by Hohle et al. (2010), based on 2MASS photometry and Hipparcos parallax. By matching temperature and surface gravity values to the range derived from spectral fitting of the LAMOST-HVS1, we infer its spectral type to be between B1 and B2.5. While the luminosity class is not wellconstrained, it is correlated with the spectral type. For example, the star could be B1I, B2IV, or B2.5V. The degeneracy leads to only a small variation in luminosity among such stars, which translates to a relatively well-constrained distance, $13.4 \pm 2.2 \mathrm{kpc}$. With $8 \mathrm{kpc}$ adopted for the Sun's distance to the GC, the Galactocentric distance of LAMOST-HVS1 is calculated to be $R=19.4 \pm 2.1 \mathrm{kpc}$. The mass of LAMOST-HVS1 is inferred to be $9.1 \pm 0.7 M_{\odot}$.

Interestingly, LAMOST-HVS1 appears to be almost a twin to HE 0437-5439 (a.k.a HVS 3; e.g., Edelmann et al. 2005; Bonanos et al. 2008; Przybilla et al. 2008), which is also a $\sim 9 M_{\odot}$ B-type star, with similar temperature and surface gravity. The Galactic position of LAMOST-HVS1 shares some similarities with HD 271791 (Heber et al. 2008), a $11 \pm 1 M_{\odot}$ B-giant star established to be a runaway star with velocity similar to those of HVSs. HD 271791 is $21.8 \pm 3.7 \mathrm{kpc}$ away from the $\mathrm{GC}$ and $-10.4 \pm 2.0 \mathrm{kpc}$ below the disk plane (Heber et al. 2008), while for LAMOST-HVS1 those are $19.4 \pm 2.1 \mathrm{kpc}$ and $7.8 \pm 1.3 \mathrm{kpc}$. Together, the above three stars make the most massive HVSs discovered so far, and LAMOST-HVS1 is the closest one.

With the velocity and distance determined, Figure 2 places LAMOST-HVS 1 in the $v_{\mathrm{rf}}-R$ plane, and compares it to the known HVSs, as well as possible HVSs and possible bound HVSs, as listed in Brown et al. (2012). Clearly, LAMOSTHVS1 is the nearest HVS discovered so far. Following Brown et al. (2012), we also plot two curves (long and short-dashed) of escape velocities, based on the Galactic potential models of Kenyon et al. (2008) and Gnedin et al. (2010), respectively. At $R \sim 20 \mathrm{kpc}$, the velocity $v_{\mathrm{rf}}=477 \mathrm{~km} \mathrm{~s}^{-1}$ of LAMOST-HVS1 is above the escape velocity in the Gnedin et al. (2010) model and falls almost on top of the model curve from Kenyon et al. (2008).

\footnotetext{
12 http://ulyss.univ-lyon1.fr/
} 


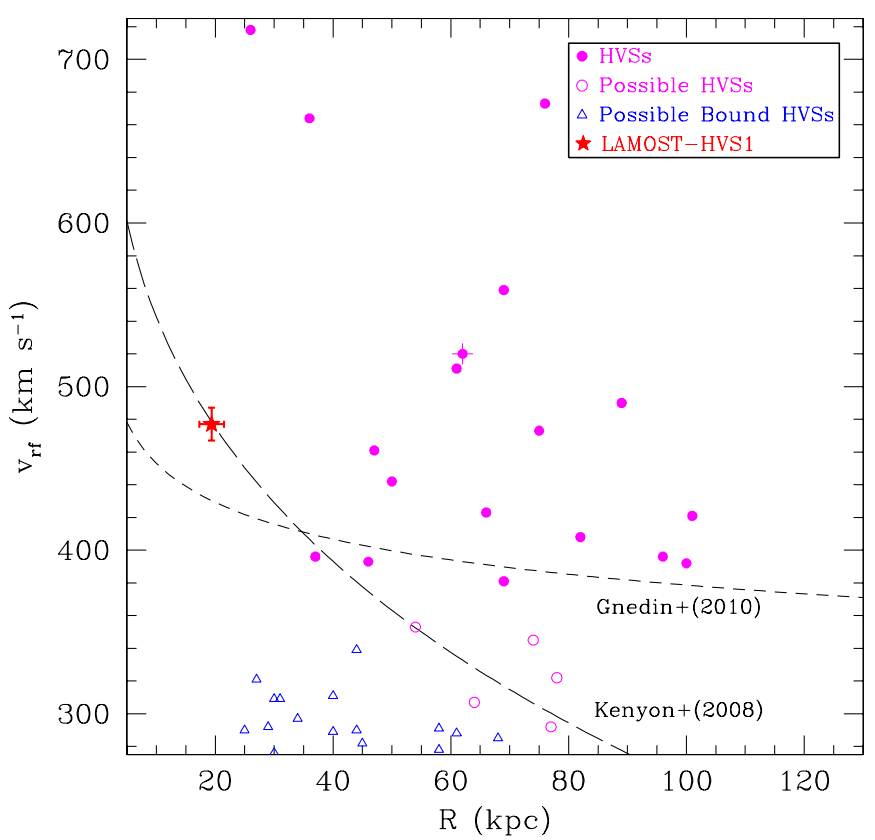

Figure 2. Galactocentric radial velocity of known/possible/bound HVSs (Brown et al. 2012) and LAMOST-HVS1 versus the Galactocentric distance. The short and dashed curves are escape velocities from two models of Galactic potential (Kenyon et al. 2008; Gnedin et al. 2010), and the difference illustrates the current uncertainties in the models. The filled circle with a cross is HVS HE 0437-5439, LAMOST-HVS1's near twin (see the text).

(A color version of this figure is available in the online journal.)

Note that including the proper motion contribution (using the PPMXL one for the most conservative estimate) implies a total velocity of $545 \pm 54 \mathrm{~km} \mathrm{~s}^{-1}$, with the uncertainties from distance, proper motion, and radial velocity. This establishes LAMOST-HVS1's identity as an HVS.

\subsection{Galactic Center Origin?}

We now explore to what extent the data constrain the origin of LAMOST-HVS1.

Lu et al. (2010) and Zhang et al. (2013) demonstrate that, under the tidal-disruption scenario, the spatial distribution of HVSs can track that of the progenitors. Lu et al. (2010) indeed find that most of the discovered HVSs, if viewed from the GC, show spatial distributions near the great circles connecting to the planes of the clockwise-rotating young stellar (CWS) disk and the northern arm of the mini-spiral or the outer wrapped part of the CWS disk, supporting the GC origin of HVSs (but see also Pawlowski et al. 2013).

Following Lu et al. (2010), the top panel of Figure 3 shows the sky distribution of the known and possible HVSs and the position of LAMOST-HVS1, as viewed from the GC. The great circles correspond to different stellar structures in the GC: the CWS, the outer wrap of the CWS (Outer-CWS), the northern arm (North-arm), and the counter-clockwise stellar disk (CCWS). Interestingly, LAMOST-HVS1 falls into the clustered region defined by most other known HVSs. It is closest to the Outer-CWS great circle and also close to that of the Northarm. This seems to support its GC origin, and suggests that its progenitors were associated with the CWS or North-arm.

In the top panel of Figure 3, LAMOST-HVS1's twin, HE 0437-5439, lies near the CCWS circle. However, it is also possible to connect to the Outer-CWS, similar to LAMOSTHVS1. HE 0437-5439 has been proposed to have been produced
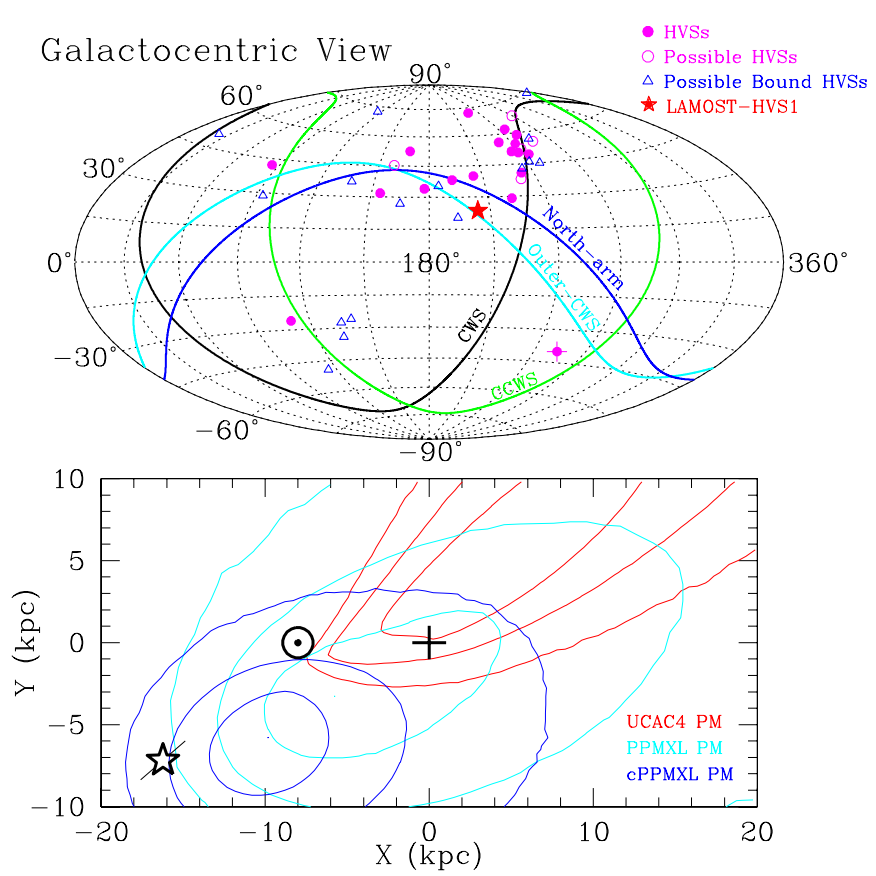

Figure 3. Top: Galactocentric view of the sky distribution of HVSs in the Galactic coordinate system and the position of LAMOST-HVS1. The positions of stars are shifted to match what would be seen by an observer at the Galactic center. The great circles correspond to structures of stellar distribution near the massive black hole at the Galactic center (see the text). The filled circle with a cross is HE 0437-5439, LAMOST-HVS1's twin (see the text). Bottom: distribution of ejection position, if LAMOST-HVS1 originated from the Galactic plane. The contours indicate the $1 \sigma, 2 \sigma$, and $3 \sigma$ ranges (color-coded for using UCAC4, PPMXL, and cPPMXL proper motions, respectively; see the text), and the loose constraint is mainly a consequence of the large uncertainty in the proper motion. The projected position of LAMOST-HVS1 is shown as the star, with the line denoting its $1 \sigma$ uncertainty. The Sun's position and the Galactic center are marked with a $\odot$ and a cross, respectively.

(A color version of this figure is available in the online journal.)

from the Large Magellanic Cloud (Edelmann et al. 2005; Bonanos et al. 2008; Przybilla et al. 2008), but with well-measured proper motion Brown et al. (2010) conclude that it is more likely a compact binary ejected from the GC, which later evolved into a blue straggler.

The proper motion of LAMOST-HVS1 is not well-measured. We perform a Monte Carlo simulation by accounting for the uncertainties in the proper motion (for the PPMXL, cPPMXL, and the UCAC4 values), distance, and radial velocity (all assumed to be Gaussian), in order to consider the implications of the velocity vector for the origin of LAMOST-HVS1. Obviously, the uncertainties are dominated by those in the proper motion.

From the star's current position, we go along the opposite direction of the velocity vector and perform a full orbit integration with the Galactic potential model in Kenyon et al. (2008) to derive the intercept position in the disk plane. This would be the point of origin for the HVS if it were ejected from the plane. The Monte Carlo simulation shows that the most likely intercept depends on which proper motion measurement is used (see the bottom panel of Figure 3). The GC is within the $\sim 1 \sigma$ $(<1 \sigma)$ range of the distribution of the intercept positions with the UCAC4 (PPMXL) proper motion adopted. If we use the PPMXL proper motion corrected for the offset of quasars, the data then favor a disk origin, with the most likely intercept lies close to the Perseus spiral arm. An accurate measurement of the proper motion will be key to determining the origin of LAMOST-HVS1. If the GC origin holds, the flight time $(\sim 32 \mathrm{Myr})$ for 
LAMOST-HVS1 to reach its current position would be comparable to its lifetime (estimated to be around 30-40 Myr). If the ejection is delayed by a few million years or more since the formation (e.g., Brown et al. 2012), there would be some tension between the delay + flight time and the lifetime. Therefore, it may be either a massive star ejected directly from the GC (with properties in broad agreements with predictions by Zhang et al. 2013), or a blue straggler from similar processes as HE 0437-5439.

We cannot rule out a disk (runaway) origin of LAMOST-HVS1, especially if we use the cPPMXL proper motion. As mentioned before, the position of LAMOST-HVS1 reminds us of the B-type giant HD 271791, which is about $10 \mathrm{kpc}$ from the disk plane. Its proper motion measurement clearly favors a disk origin, establishing it as a hyper-velocity runaway star (Heber et al. 2008). Bromley et al. (2009) show that high speed stars near the disk should mostly be disk runaway stars, and HD 271791 is therefore an example of unbound runaway stars. For LAMOST-HVS1, a disk runaway origin would greatly alleviate the potential tension between flight time and the lifetime of the star as in the GC-origin scenario. A better proper motion determination for LAMOST-HVS1 will help to show whether the possibility of a disk runaway star holds.

Given that LAMOST-HVS1 is the brightest example of known HVSs and its clear similarity to HE 0437-5439 and HD 271791, it will be extremely interesting to conduct a more detailed study. If indeed it comes from the GC, we expect a

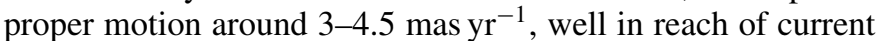
and near future data. High-resolution spectroscopic study will reveal its chemical abundance pattern and rotation velocity. We plan to perform such follow-up observations and investigations.

\section{SUMMARY AND DISCUSSION}

We present the first HVS discovered from the LAMOST survey, a B-type star with a mass of $\sim 9 M_{\odot}$, located at a Galactocentric distance of $\sim 19 \mathrm{kpc}$ with a Galactocentric radial velocity component of $\sim 477 \mathrm{~km} \mathrm{~s}^{-1}$, based on the measured radial velocity. It is the closest and one of the three most massive HVSs discovered so far.

LAMOST-HVS1 is clustered with most other known HVSs on the sky. Its proximity to the great circles corresponding to stellar structures around the central MBH suggests a GC origin. A more accurate proper motion measurement, achievable in the near future, will pin down its origin.

LAMOST-HVS1 signals the start of the HVS discovery effort from the LAMOST survey. The survey has the potential to discover a large number of HVSs. Figure 4 shows a conservative forecast with a Monte Carlo method for the plan of a 16,000 deg ${ }^{2}$ survey with a limiting magnitude of $r=17.5$ (the final survey may be deeper), following a LEGUE halo target selection with higher priority on bluer stars (Carlin et al. 2012). The forecast assumes a GC origin of HVSs and is normalized by using the space density $0.077(R / \mathrm{kpc})^{-2} \mathrm{kpc}^{-3}$ of $3 M_{\odot}<M<4 M_{\odot}$ HVSs in Brown et al. (2007b). A maximum mass of $10 M_{\odot}$ is adopted in computing the cumulative count. Three cases are considered: a Salpeter mass function (MF; Salpeter 1955), a Galactic bulge MF (Mezger et al. 1999), and a GC MF (Lu et al. 2013). The forecast implicitly assumes that in the mass range considered, stars have identical binary fractions, distribution of binary orbital separations, and $\mathrm{MBH}$ ejection velocities, which may not be true in detail but serves our estimation purpose.

Since the LAMOST survey does not preselect B-type stars as targets for spectroscopic observations to reduce the

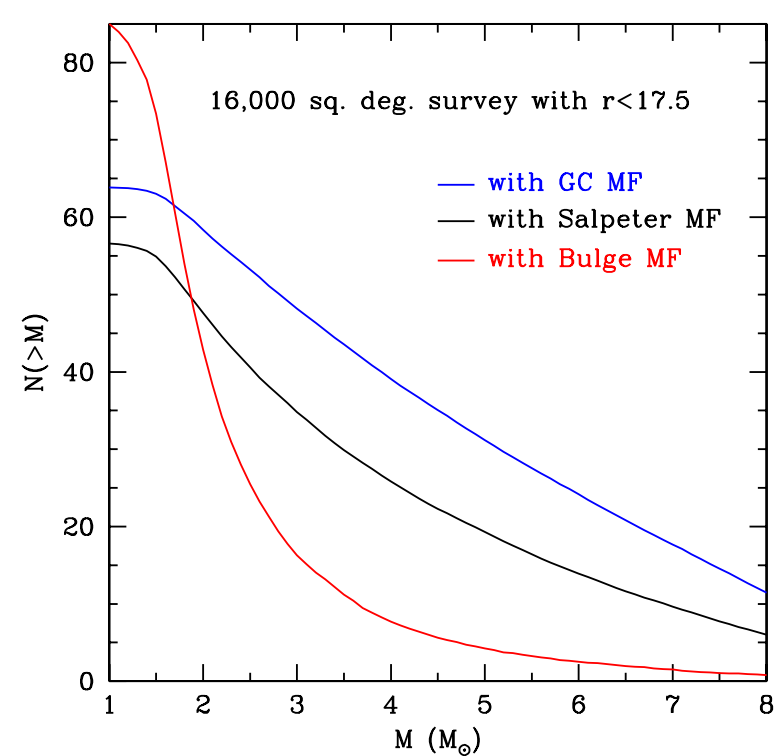

Figure 4. Prospect of discovering (unbound) HVSs from the LAMOST survey, based on the plan of a 16,000 deg ${ }^{2}$ survey with a limiting magnitude $r=17.5$. The curves represent the numbers of HVSs that LAMOST may discover for three cases of the stellar MF.

(A color version of this figure is available in the online journal.)

contamination by the large number of halo stars, it can discover HVSs of various stellar types, from B to $G$. The total number of HVSs down to $M=1 M_{\odot}$ with the above survey parameters is in the range of $\sim 56-85$. In addition, we also expect to find a large number of bound HVSs and possibly binary HVSs. Such a large statistical sample of HVSs of different types would enable a wide range of investigations to elucidate the nature of HVSs, and to constrain Galactic structure.

Z.Z. thanks Youjun Lu, Qingjuan Yu, and Ben Bromley for helpful discussions. We thank the referee for a prompt and constructive report. This work is supported by the U.S. National Science Foundation (NSF), through grant AST-0937523. T.C.B. acknowledges partial support from U.S. NSF grant PHY 0822648. P.G. thanks the U.S. NSF for grant AST-1010039. Guoshoujing Telescope (the Large Sky Area Multi-Object Fiber Spectroscopic Telescope LAMOST) is a National Major Scientific Project built by the Chinese Academy of Sciences. Funding for the project has been provided by the National Development and Reform Commission. LAMOST is operated and managed by the National Astronomical Observatories, Chinese Academy of Sciences.

\section{REFERENCES}

Abadi, M. G., Navarro, J. F., \& Steinmetz, M. 2009, ApJL, 691, L63

Bonanos, A. Z., López-Morales, M., Hunter, I., \& Ryans, R. S. I. 2008, ApJL, 675, L77

Blaauw, A. 1961, BAN, 15, 265

Bromley, B. C., Kenyon, S. J., Brown, W. R., \& Geller, M. J. 2009, ApJ, 706,925

Bromley, B. C., Kenyon, S. J., Geller, M. J., \& Brown, W. R. 2012, ApJL, 749, L42

Brown, W. R., Anderson, J., Gnedin, O. Y., et al. 2010, ApJL, 719, L23

Brown, W. R., Geller, M. J., Kenyon, S. J., \& Kurtz, M. J. 2005, ApJL, 622, L33

Brown, W. R., Geller, M. J., Kenyon, S. J., \& Kurtz, M. J. 2006a, ApJL, 640, L35

Brown, W. R., Geller, M. J., Kenyon, S. J., \& Kurtz, M. J. 2006b, ApJ, 647, 303

Brown, W. R., Geller, M. J., Kenyon, S. J., Kurtz, M. J., \& Bromley, B. C. 2007a, ApJ, 660, 311 
Brown, W. R., Geller, M. J., Kenyon, S. J., Kurtz, M. J., \& Bromley, B. C. 2007b, ApJ, 671, 1708

Brown, W. R., Geller, M. J., \& Kenyon, S. J. 2012, ApJ, 751, 55

Carlin, J. L., Lépine, S., Newberg, H. J., et al. 2012, RAA, 12, 755

Chen, L., Hou, J.-L., Yu, J.-C., et al. 2012, RAA, 12, 805

Cui, X.-Q., Zhao, Y.-H., Chu, Y.-Q., et al. 2012, RAA, 12, 1197

Deng, L.-C., Newberg, H. J., Liu, C., et al. 2012, RAA, 12, 735

Edelmann, H., Napiwotzki, R., Heber, U., Christlieb, N., \& Reimers, D. 2005, ApJL, 634, L181

Gnedin, O. Y., Brown, W. R., Geller, M. J., \& Kenyon, S. J. 2010, ApJL, 720, L108

Gnedin, O. Y., Gould, A., Miralda-Escudé, J., \& Zentner, A. R. 2005, ApJ, 634,344

Gvaramadze, V. V., Gualandris, A., \& Portegies Zwart, S. 2009, MNRAS, 396,570

Heber, U., Edelmann, H., Napiwotzki, R., Altmann, M., \& Scholz, R.-D. 2008, A\&A, 483, L21

Hills, J. G. 1988, Natur, 331, 687

Hirsch, H. A., Heber, U., O’Toole, S. J., \& Bresolin, F. 2005, A\&A, 444, L61

Hohle, M. M., Neuhäuser, R., \& Schutz, B. F. 2010, AN, 331, 349

Kenyon, S. J., Bromley, B. C., Geller, M. J., \& Brown, W. R. 2008, ApJ, 680,312

Koleva, M., Prugniel, Ph., Bouchard, A., \& Wu, Y. 2009, A\&A, 501, 1269

Kollmeier, J. A., \& Gould, A. 2007, ApJ, 664, 343
Kollmeier, J. A., Gould, A., Rockosi, C., et al. 2010, ApJ, 723, 812

Lu, J. R., Do, T., Ghez, A. M., et al. 2013, ApJ, 764, 155

Lu, Y., Yu, Q., \& Lin, D. N. C. 2007, ApJL, 666, L89

Lu, Y., Zhang, F., \& Yu, Q. 2010, ApJ, 709, 1356

Luo, A.-L., Zhang, H.-T., Zhao, Y.-H., et al. 2012, RAA, 12, 1243

McMillan, P. J., \& Binney, J. J. 2010, MNRAS, 402, 934

Mezger, P. G., Zylka, R., Philipp, S., \& Launhardt, R. 1999, A\&A, 348,457

Pawlowski, M. S., Kroupa, P., \& Jerjen, H. 2013, MNRAS, 435, 1928

Przybilla, N., Nieva, M. F., Heber, U., et al. 2008, A\&A, 480, L37

Reid, M. J., Menten, K. M., Zheng, X. W., et al. 2009, ApJ, 700, 137

Roeser, S., Demleitner, M., \& Schilbach, E. 2010, AJ, 139, 2440

Salpeter, E. E. 1955, ApJ, 121, 161

Schönrich, R., Binney, J., \& Dehnen, W. 2010, MNRAS, 403, 1829

Tutukov, A. V., \& Fedorova, A. V. 2009, ARep, 53, 839

Wang, B., \& Han, Z. 2009, A\&A, 508, L27

Yang, F., Carlin, J. L., Liu, C., et al. 2012, RAA, 12, 781

Yu, Q., \& Madau, P. 2007, MNRAS, 379, 1293

Yu, Q., \& Tremaine, S. 2003, ApJ, 599, 1129

Zacharias, N., Finch, C. T., Girard, T. M., et al. 2013, AJ, 145, 44

Zhang, F., Lu, Y., \& Yu, Q. 2013, ApJ, 768, 153

Zhang, Y.-Y., Carlin, J. L., Yang, F., et al. 2012, RAA, 12, 792

Zhao, G., Zhao, Y.-H., Chu, Y.-Q., Jing, Y.-P., \& Deng, L.-C. 2012, RAA, 12,723 